\title{
Transglutaminase in Receptor and Neurotransmitter-Regulated Functions
}

\author{
Nancy A. Muma \\ Department of Pharmacology and Toxicology, University of Kansas School \\ of Pharmacy, 5064 Malott Hall, 1251 Wescoe Hall Drive, Lawrence, Kansas
} 66045, USA. Email: nmuma@ku.edu.

\begin{abstract}
Transglutaminases (TGs) and especially TG2 play important roles in neurotransmitter and receptor signaling pathways. Three different mechanisms by which TG2 interacts with neurotransmitter and receptor signaling systems will be discussed in this review. The first way in which TG2 interacts with receptor signaling is via its function as a guanine nucleotide binding protein (G-protein) coupling to G-protein coupled receptors (GPCRs) to activate down-stream signaling pathways. TG2 can exist in a least two conformations, a closed GTP-bound conformation and an open calcium-bound conformation. In the closed GTP-bound conformation, TG2 is capable of functioning as a G-protein for GPCRs. In the open calcium-bound conformation, TG2 catalyzes a transamidation reaction cross-linking proteins or catalyzing the covalent binding of a mono- or polyamine to a protein. The second mechanism is regulation of the transamidation reaction catalyzed by TG2 via receptor stimulation which can increase local calcium concentrations and thereby increase transamidation reactions. The third way in which TG2 plays a role in neurotransmitter and receptor signaling systems is via its use of monoamine neurotransmitters as a substrate. Monoamine neurotransmitters including serotonin can be substrates for transamidation to a protein often a small G-protein (also known as a small GTPase) resulting in activation of the small G-protein. The transamidation of a monoamine neurotransmitter or serotonin has been designated as monoaminylation or more specifically serotonylation, respectively. Other proteins are also targets for monoaminylation such as fibronectin and cytoskeletal proteins. These receptor and neurotransmitter-regulated reactions by TG2 play roles in physiological and key pathophysiological processes.
\end{abstract}

\section{AOPEN ACCESS}

DOI: $10.20900 / \mathrm{mo} .20180012$

Received: October 30, 2018

Accepted: November 28, 2018

Published: December 5, 2018

Copyright: () 2018 by the author. Licensee Hapres, London, United Kingdom. This is an open access article distributed under the terms and conditions of the Creative Commons Attribution License (CC BY 4.0, https://creativecommons.org/licenses/by/4.0/).

Keywords: serotonylation; monoaminylation; transglutaminase; transamidation; Gah

\section{INTRODUCTION}

Transglutaminases (TGs) (EC2.3.2.13) are known to play diverse roles in normal physiological functions as well as pathological conditions, from blood clot formation ${ }^{[1,2]}$ and stabilization of hair and epidermis ${ }^{[3]}$ to 
cancer $^{[4]}$ and celiac disease ${ }^{[5]}$. More recent studies have begun to uncover essential roles for TGs in neurotransmitter and receptor signaling-related functions. This review will examine our current understanding of the roles of TGs in receptor and neurotransmitter signaling and highlight opportunities for further exploration.

TGs are a family of structurally and functionally related enzymes that catalyze modifications to peptidebound glutamine residues including transamidation, deamidation and esterification ${ }^{[6]}$. These enzymes can catalyze a transamidation reaction wherein a covalent bond is formed between a peptide-bound glutamine and a primary amine. The primary amine can be a monoamine such as serotonin, a polyamine such as putrescine, or donated by a lysine residue in a polypeptide chain or protein. The TG-catalyzed isopeptide linkage [NE-( $\mathrm{Y}$-l-glutamyl)-I-lysine] or so called cross-link between peptide-bound lysine and peptide-bound glutamine residues can occur within a single polypeptide chain or between two polypeptide chains. Deamidation occurs when $\mathrm{H}_{2} \mathrm{O}$ reacts with a peptide-bound glutamine in place of a primary amine ${ }^{[7]}$, while esterification occurs when an alcohol reacts with glutamine ${ }^{[3]}$.

There are nine genes that code for human TGs, eight of which encode catalytically active enzymes ${ }^{[8]}$. These active enzymes include TG 1-7 and factor XIIla. In general, the distribution of these enzymes is limited to particular tissues. Unlike other TGs, TG2 is ubiquitously expressed in tissues and found both within cells and in the extracellular space. Within cells, TG2 is found in the cell membrane, cytoplasm, nucleus and mitochondria.
TG2 is also unique among TG proteins in that in addition to its function as an enzyme that can catalyze transamidation of proteins, TG2 is a multifunctional protein capable of guanosine triphosphatase (GTPase) and adenosine triphosphatase (ATPase) activity, activity as a kinase and protein disulfide isomerase, and has cell adhesion and scaffolding functions ${ }^{[8,9]}$.

\section{TG AS A G-PROTEIN FOR GPCRS}

Over thirty years ago, TG2 was first shown to bind GTP and inhibit the transamidation activity of TG ${ }^{[10]}$. Subsequently, TG2 was shown to function as a guanosine triphosphate binding protein (G-protein) for the transduction of signals for GPCRs ${ }^{[11]}$. In this capacity as a G-protein, TG2 is also known as Gah. TG2 can exist in at least two different conformations depending whether it's bound to $\mathrm{Ca}^{2+}$ or the nucleotides GTP or ATP, although the affinity for GTP is much higher than for ATP ${ }^{[12]}$. In the GTP-bound conformation, TG2 takes on a closed conformation and functions as a GTPase ${ }^{[9]}$. While in the $\mathrm{Ca}^{2+}$-bound conformation, TG2 takes on an open conformation capable of catalyzing transamidation reactions (Fig. 1). In the closed, GTP-bound conformation, TG2 is capable of mediating the activation of phospholipase C (PLC) $\Delta 1$ following stimulation of $\alpha 1$ adrenoreceptors ${ }^{[11,13]}$, oxytocin receptors ${ }^{[14,15]}$, thromboxane $A 2 \alpha^{[16]}$, and follicle-stimulating hormone receptors ${ }^{[17]}$. At this time, it is unclear how widespread the use of TG2/Gah is for GPCR signal transduction and how many different GPCRs can use TG2/Gah for signal transduction.

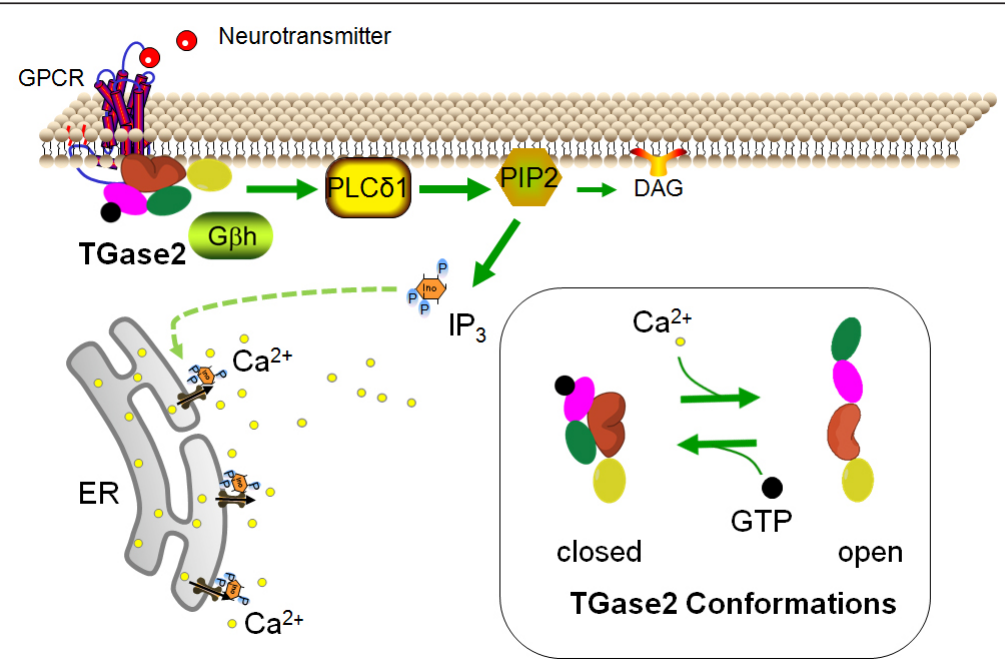

Fig. 1 TG2 in the closed conformation. In the closed conformation, TG2 functions as a G-protein capable of coupling to GPCRs and signaling through down-stream pathways including activation of PLC 1 to convert PIP2 to $D A G$ and $I P_{3}$. IP 3 binds to $\mathbb{I P}_{3}$ receptors on the ER leading to the release of $\mathrm{Ca}^{2+}$ from the ER. In the GTPbound form, TG2/Gah dissociates from calreticulin/Gßh. Insert: TG2 adopts a closed conformation and binds GTP (small black circle) in the presence of low $\mathrm{Ca}^{2+}$ and high GTP concentrations. In higher concentrations of $\mathrm{Ca}^{2+}$, TG2 binds $\mathrm{Ca}^{2+}$ and adopts an open conformation. The four domains of TG2 are depicted as yellow, orange, pink and green ovals to represent the $\beta$-sandwich, catalytic core, $\beta$-barrel 1 and $\beta$-barrel 2 domains. 
GPCRs are classically known to couple to heterotrimeric G-proteins consisting of a GTP or GDP-bound $G a$ subunit and tightly associated $G \beta$ and $G y$ subunits. In contrast, TG2/Gah functions as a heterodimeric G-protein with calreticulin functioning as the $\mathrm{G} \beta \mathrm{h}$ protein ${ }^{[18,19]}$. The functioning of the heterodimeric TG2/Gah and calreticulin/ $\mathrm{G} \beta \mathrm{h}$ is similar to the heterotrimeric G-proteins. Receptor activation facilitates the dissociation of GDP from TG2/Gah followed by association of GTP. Calreticulin/Gßh then dissociates from TG2/Gah. Signaling by TG2/Gah is terminated by hydrolysis of GTP-bound to TG2/Gah and re-association of

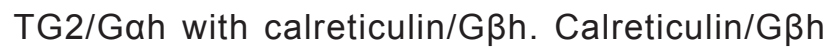
associates with the GDP-bound form of TG2/Gah and inhibits the binding of TG2/Gah to GTP as well as inhibiting the transamidation activity of TG2 ${ }^{[18]}$.

\section{RECEPTOR ACTIVATION OF TG-CATALYZED TRANSAMIDATION}

Receptor stimulation plays an important role in regulating TG-catalyzed transamidation via altering $\mathrm{Ca}^{2+}$ concentrations in the micro-environment. The ability of TG to catalyze transamidation reactions depends on the surrounding concentrations of ATP,
GTP and $\mathrm{Ca}^{2+}$. Smethurst and Griffin ${ }^{[20]}$ estimated that in the cell cytosol the concentration of ATP is approximately $8 \pm 11 \mathrm{nM}$ and GTP is $50 \pm 300 \mu \mathrm{M}$ with free GTP at about $100 \mu \mathrm{M}$. The cytoplasmic $\mathrm{Ca}^{2+}$ concentration averages about $100 \mathrm{nM}$. In contrast, the extracellular $\mathrm{Ca}^{2+}$ concentration is about $2 \mathrm{mM}$. The large difference in $\mathrm{Ca}^{2+}$ concentrations is maintained by sequestering $\mathrm{Ca}^{2+}$ in the endoplasmic reticulum (ER) and mitochondria, $\mathrm{Ca}^{2+}$ pumps and exchangers that move $\mathrm{Ca}^{2+}$ out of the cell and binding to one of hundreds of $\mathrm{Ca}^{2+}$ binding proteins ${ }^{[21]}$. It is estimated that in the presence of $100 \mu \mathrm{M}$ GTP, $100 \mu \mathrm{M}$ of $\mathrm{Ca}^{2+}$ is necessary for transamidation by TG2 ${ }^{[20]}$. These estimates suggest that unless the local concentration of $\mathrm{Ca}^{2+}$ is increased, transamidation reactions will not be catalyzed by TG2 within cells.

That's where receptor signaling comes into play. $\mathrm{Ca}^{2+}$ concentrations can be increased in the micro-environment following the stimulation of Gaq/11-coupled GPCRs or neurotransmitter-regulated ion channels (Fig. 2). Stimulation of Gaq/11-coupled GPCRs leads to activation of PLC $\beta$ which catalyzes the formation of diacylglycerol (DAG) and inositol 1,4,5-triphosphate $\left(\mathrm{IP}_{3}\right)$ from phosphatidylinositol 4,5-biphosphate (PIP2). $\mathrm{IP}_{3}$ can bind to $\mathrm{IP}_{3}$ receptors on the ER leading to the release of $\mathrm{Ca}^{2+}$, increasing $\mathrm{Ca}^{2+}$ concentrations to about $1 \mu \mathrm{M}$ for many seconds ${ }^{[21]}$. This concentration of $\mathrm{Ca}^{2+}$ is theoretically sufficient to allow TG2 to catalyze transamidation reactions.

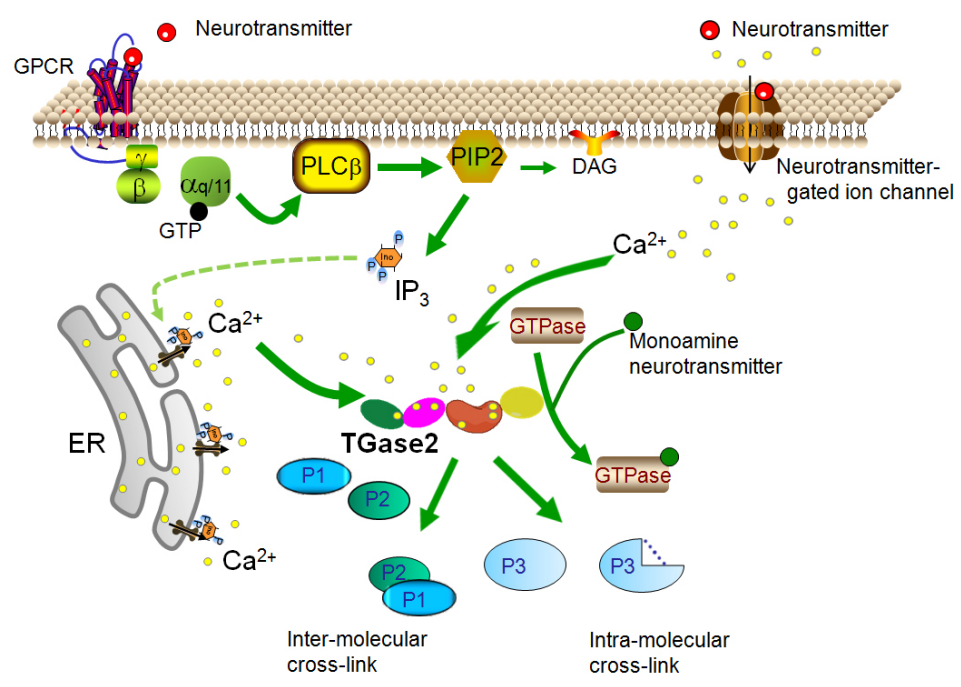

Fig. 2 TG2 in the open conformation. In the open conformation, TG2 catalyzes transamidation reactions. In human TG2, there are six binding sites for $\mathrm{Ca}^{2+}$ ions (small yellow circles) to bind to TG2 in the open conformation. These $\mathrm{Ca}^{2+}$ binding sites are highly conserved attesting to their importance ${ }^{[22]}$. Mutations within these binding sites can give rise to reduced transamidation activity and are found in patients with disorders in glucose metabolism ${ }^{[23]}$. The elevation of $\mathrm{Ca}^{2+}$ concentrations needed for the open conformation can occur at the cell membrane through two well defined neurotransmitter-dependent mechanisms. $\mathrm{Ca}^{2+}$ concentrations can be elevated at the cell membrane through neurotransmitter-gated ion channels (top right) or through Gaq/11-coupled GPCRs (upper left) activation of PLC $\beta$ and production of $\mathrm{IP}_{3}$. Stimulation of $\mathrm{IP}_{3}$ receptors on the ER results in release of $\mathrm{Ca}^{2+}$ (lower left). The TG2-catalyzed transamidation reaction can result in intermolecular cross-linked proteins, intramolecular cross-linked proteins or transamidation of poly- or monoamines to proteins such as monoaminylation of small G-proteins (GTPases). A third receptormediated mechanism to increase TG2 transamidation appears to involve the transport of extracellular $\mathrm{Ca}^{2+}$ into the cell but is less well understood and not shown here. 
Importantly, increased transamidation has been demonstrated following the stimulation of several Gaq/11-coupled GPCRs ${ }^{[24]}$ and has been shown to be dependent on the increase in intracellular $\mathrm{Ca}^{2+[25]}$. Muscarinic 3 (M3) receptors are Gaq/11-coupled GPCRs that lead to the production of $\mathrm{IP}_{3}$ and release of $\mathrm{Ca}^{2+}$ from ER. In differentiated SH-SY5Y cells (a neuroblastoma cell line) stimulation of muscarinic receptors with carbachol and inhibition of $\mathrm{Ca}^{2+}$ uptake by the ER with thapsigargin both resulted in increased transamidation activity ${ }^{[26]}$. Stimulation of serotonin (5-HT) 2A receptors in a rat cortical cell line ${ }^{[27]}$, $5-\mathrm{HT} 2 \mathrm{~A}$ and $5-\mathrm{HT} 2 \mathrm{C}$ receptors in primary rat cortical cells and muscarinic receptors in SH-SY5Y cells were each shown to increase transamidation of monoamines to small G-proteins ${ }^{[24]}$. To determine if increases in local $\mathrm{Ca}^{2+}$ concentrations and PLCcatalyzed production of $\mathrm{IP}_{3}$ following stimulation of Gaq/11-coupled GPCRs was necessary to increase transamidation, PLC inhibitors and $\mathrm{Ca}^{2+}$ chelation were used. Indeed, the increase in transamidation following stimulation of 5-HT2A receptors was shown to be dependent on PLC activation and an increase in intracellular $\mathrm{Ca}^{2+}$ concentrations ${ }^{[25]}$. Furthermore, the increase in intracellular $\mathrm{Ca}^{2+}$ concentrations was found to be sufficient to increase transamidation as treatment with the $\mathrm{Ca}^{2+}$ ionophore ionomycin increased transamidation independently of receptor stimulation ${ }^{[25]}$.

Local $\mathrm{Ca}^{2+}$ concentrations at the cell membrane can also be increased following stimulation of neurotransmitter-gated ion channels and so could thereby increase TG2-mediated transamidation. Stimulation of N-methyl-D-aspartate (NMDA) receptors causes an influx of $\mathrm{Ca}^{2+}$, transiently increasing local $\mathrm{Ca}^{2+}$ concentrations resulting in increased transamidation in a mouse neuroblastoma cell line, Neuro2a cells ${ }^{[15]}$. An increase in transamidation has not been demonstrated for other neurotransmittergated $\mathrm{Ca}^{2+}$ channels but should be examined.

A third receptor-mediated mechanism can result in increased TG2-catalyzed transamidation activity. $\beta 2$-adrenoreceptor ${ }^{[28]}$ and $A_{1}$ adenosine receptor ${ }^{[29]}$ activation can increase TG2 transamidation activity and the increase is at least partially dependent on an influx of extracellular $\mathrm{Ca}^{2+}$. These GPCRs are Gai/o-coupled GPCRs, not Gaq/11-coupled that could increase intracellular $\mathrm{Ca}^{2+}$ via down-stream $\mathrm{IP}_{3}$ receptor stimulation. $\mathrm{Ca}^{2+}$ influx following receptor stimulation was shown to be necessary for the increase in transamidation following stimulation of the Gai/o-coupled GPCRs. In addition to regulation of transamidation activity via a $\mathrm{Ca}^{2+}$ influx, the increased transamidation activity was also dependent on activation of several kinases, specifically protein kinase $A(P K A)$, extracellular signal-regulated kinase 1/2 (ERK1/2) and phosphoinositol 3-kinase (PI-3K) for $\beta 2$-adrenoreceptor-mediated transamidation activation and PKC, mitogen-activated kinase kinase (MEK) 1/2 and c-Jun N-terminal kinase (JNK) 1/2 for $\mathrm{A}_{1}$ adenosine receptors. It was also noted in those studies that TG2 was phosphorylated following receptor activation and that inhibition of these kinases prevented the receptor induced-phosphorylation of TG2. However, these enzymes had no direct effect on TG activity in vitro.

$\mathrm{PAC}_{1}$ receptor activation also increases $\mathrm{TG} 2$ transamidation activity ${ }^{[30]}$. $\mathrm{PAC}_{1}$ receptors are Gas and Gaq/11-linked GPCRs. However, similar to the Gai/o-coupled $\beta 2$-adrenoreceptor and $A_{1}$ adenosine receptor-activation of TG2, transamidation is dependent on an influx of extracellular $\mathrm{Ca}^{2+}$ and kinase signaling pathways specifically PKA, PKC and MEK1/2. Phosphorylation of TG2 was also associated with activation of $\mathrm{PAC}_{1}$ receptors. Although it is not known whether TG2 phosphorylation is required for increased transamidation activity or whether TG2 phosphorylation is the mechanism by which these kinases activate TG2, the correlation suggests that it is possible and should be further investigated. It is also possible that kinase activity increases transamidation activity via increases in local $\mathrm{Ca}^{2+}$ concentrations, via altering protein-protein interactions which can lower the concentration of $\mathrm{Ca}^{2+}$ needed for transamidation ${ }^{[31]}$ or more directly via altering the substrate conformation. In contrast, PKA phosphorylation of TG2 in mouse embryonic fi roblasts inhibited in vitro transamidation activity and increased kinase activity ${ }^{[32]}$. Further studies are needed to elucidate the mechanisms by which phosphorylation and receptor-mediated kinase activity impact TG2 transamidation activity.

A novel and clever Förster resonance energy transfer (FRET) approach was developed whereby the amino and carboxyl terminals of TG2 are tagged with yellow and cyan fluorescence proteins to monitor the protein conformation ${ }^{[33]}$. In the closed conformation, the amino and carboxyl terminals are in close proximity which can produce a FRET signal from the tagged construct. However, the terminals are quite distant in the open conformation, and thereby not capable of producing a FRET signal. TG2 located near the cell membrane was found to be predominantly in the open conformation capable of catalyzing the transamidation reaction ${ }^{[33]}$. In contrast, much of the remaining cytoplasm contained TG2 in the closed conformation. Since TG2 is already in the open $\mathrm{Ca}^{2+}$ bound conformation, how would an increase in $\mathrm{Ca}^{2+}$ following receptor stimulation cause an increase in transamidation? These FRET data may at first appear to contradict the findings that receptormediated increases of $\mathrm{Ca}^{2+}$ at the cell membrane are needed for transamidation. However, it is also possible that under the cell culture conditions used in this study, sufficient levels of receptor activation resulted in high levels of $\mathrm{Ca}^{2+}$ at the cell membrane. 
In the FRET experiments, the cells were cultured in the standard $10 \%$ fetal bovine serum which would likely contain numerous neurotransmitters capable of receptor activation leading to local increases in $\mathrm{Ca}^{2+}$ concentrations. Indeed, in other cell lines, the use of charcoal-filtered serum in the cell culture media is necessary to remove monoamines and other neurotransmitters to demonstrate serotonin receptor-mediated effects including increases in transamidation ${ }^{[24,25]}$. Serum-deprived cell culture conditions are usually used in studies prior to receptor stimulation and indeed in the same A549 cell line used to demonstrate TG2 conformation with FRET ${ }^{[33]}$, both charcoal-filtered and reduced serum levels in the media were used to demonstrate changes in receptor signaling ${ }^{[34]}$. Further studies using this novel FRET construct and a variety of cell culture and receptor stimulation conditions would provide excellent data to further elucidate these important phenomena.

\section{MONOAMINE NEUROTRANSMITTERS AS SUBSTRATES FOR TG}

Although we have long been aware of the transamidation of proteins to poly- and monoamines ${ }^{[7]}$, the functional impact of monoamine neurotransmitters as substrates for TG is a more recently discovered phenomenon and we are only beginning to appreciate the extent and importance of this reaction. Interest in the use of a monoamine neurotransmitters as a substrate for TGs re-emerged in 2002 with two studies describing serotonylation in platelets ${ }^{[1,35]}$. Extracellular proteins such as fibrinogen and von Willebrand factor on platelets are bound to serotonin by TGs ${ }^{[35]}$. Diego Walther and colleagues discovered that TG catalyzes the intracellular transamidation of serotonin to small G-proteins during aggregation of activated platelets resulting in activation of small G-proteins ${ }^{[1]}$. The term serotonylation was coined to describe the transamidation of serotonin to a substrate protein. As other monoamine neurotransmitters were subsequently found to be substrates for transamidation reactions the term monoaminylation was introduced ${ }^{[36,37]}$. These early studies identified the types of proteins that were later found to be common substrates for monoaminylation, namely small G-proteins and extracellular proteins. Intracellular monoaminylation is in part dependent on the availability of monoamine neurotransmitters and the presence and activity neurotransmitter transporters. This topic was recently reviewed ${ }^{[38]}$ and will not be included in this review.

Small G-proteins, also known as small GTPases, are a major target for monoaminylation. The Ras superfamily of small G-proteins can be divided into five subfamilies based on their structure and function, namely Rho, Rab, Ras, Ran and Arf ${ }^{[39]}$. To date, monoaminylation has been demonstrated for multiple proteins in the Rho and Rab subfamilies. Small G-proteins have high affinity for both GTP and GDP. Similar to heterotrimeric G-proteins, small G-proteins act as molecular switches which can bind and activate effector molecules in the GTP-bound form but not in the GDP-bound form. However, unlike most heterotrimeric G-proteins, the small G-proteins have low intrinsic GTP hydrolysis activity and rely on GTPase-activating proteins (GAPs) to hydrolyze GTP to GDP for inactivation. Guanine-nucleotide exchange factors (GEFs) play the role of activating the small G-proteins by promoting the exchange of GTP for GDP similar to GPCR stimulation for heterotrimeric G-proteins.

For the Rho family of small G-proteins, monoaminylation acts by modifying a key glutamine residue in the switch 2 region necessary for GAPstimulated hydrolysis of GTP. Since the GTPase activity of small G-proteins is exceedingly slow, this GAP-stimulated hydrolysis is required to terminate activity and maintain normal physiological function. Furthermore, monoaminylated Rho small G-proteins appear to remain constitutively active until they are degraded. Support for this mechanism comes from several lines of research on members of the Rho family small G-proteins, Rac1, Cdc42 and RhoA. First, Rac1 and Cdc42 at Q61 and RhoA at Q63 are in the switch 2 region of the small G-proteins and this glutamine is essential for GTP hydrolysis ${ }^{[40]}$. Monoaminylation of Rac1 by TG2 in neuronal cells takes place at Q61 as demonstrated by site directed mutagenesis; replacing lysine 61 with an asparagine (Rac1Q61N) prevents monoaminylation of Rac1 and surprisingly results in a constitutively active protein ${ }^{[24]}$. The constitutive activation of Rac1Q61N is likely related to the constitutive activation seen following deamidation of Rac1. Deamidation of Rac1 and Cdc42 at Q61 and RhoA at Q63 by bacterial transglutaminase results in constitutive activity of these Rho family of small G-proteins ${ }^{[41-43]}$. Serotonylation of RhoA in pulmonary artery smooth muscle cells results in constitutive activation and subsequent proteosomal degradation of RhoA ${ }^{[44]}$. In a corresponding manner, deamidation of Rac1 and Cdc42 at Q61 increases ubiquitin-mediated proteosomal degradation following activation ${ }^{[45]}$. The importance of tight physiological regulation of the Rho family of small G-proteins via modification of Q61/Q63 is demonstrated by the ability of two different bacterially produced toxins to catalyze deamidation and monoaminylation of RhoA, Rac1 and $\mathrm{Cdc} 42^{[41,42,45-47]}$.

It is not entirely clear if monoaminylation works through a similar mechanism of action to increase the activity of other small G-proteins. There is a 
conserved glutamine residue in the switch 2 region which is involved in GTP hydrolysis of many but not all small G-proteins ${ }^{[48]}$, suggesting that those small G-proteins that do rely on the switch 2 glutamine for hydrolysis could be rendered constitutively active by monoaminylation. To date several small G-proteins in the Rho and Rab families have reported to be activated by monoaminylation. There is also one report documenting serotonylation of Ras protein, the name sake for the Ras family of small G-proteins ${ }^{[49]}$. The switch 2 glutamine is present in Rab small G-proteins but is not directly involved in GTP hydrolysis ${ }^{[48]}$. However, due to the proximity of the switch 2 glutamine to the active site for hydrolysis in Rab proteins, it is possible that monoaminylation of the switch 2 glutamine could disrupt the conformation necessary for GTP hydrolysis. Indeed for Rab4, mutation of the switch 2 glutamine renders the protein constitutively active and is the site at which monoaminylation occurs and results in constitutive activation ${ }^{[50]}$. Further research is needed to determine the glutamine residues in other Rab proteins that are monoaminylated and demonstrate whether a similar mechanism is involved in their activation by monoaminylation.

Monoaminylation of the Rab family of small G proteins regulates a broad range of physiological functions. Rab proteins regulate trafficking of proteins between organelle for endocytosis and secretion and for intracellular vesicular transport ${ }^{[39]}$. Serotonylation of Rab3a and Rab27a was shown to be important for glucose0-stimulated insulin secretion from pancreatic beta cells ${ }^{[51]}$. This serotonylation of Rab3a and Rab27a results in their constitutive activation and subsequent enhancement of proteosomal degradation. Furthermore, mutations in TG2 are associated with type-2 diabetes, suggesting that abnormal serotonylation of these Rab proteins could be involved in the perturbed insulin release ${ }^{[23,52]}$. However, deletion or constitutive activation of TG2 had no effect on insulin or glucose challenge suggesting that TG2 does not regulate insulin secretion at least in mice ${ }^{[53]}$.

Serotonylation of Rab4 as mentioned above results in constitutive activation by stabilizing the active GTP-bound form of Rab4 ${ }^{[50]}$. Serotonylation of Rab4 occurs on Q67 in the switch 2 region and similar to Rac1, the Rab4Q67L mutant is constitutively active. Serotonylation of Rab4 induces binding to the serotonin transporter (SERT) and prevents the translocation of SERT to the cell membrane. SERT is a high affinity and low capacity transport mechanism moving serotonin across the cell membrane. SERT is expressed in a variety of cells and tissues in addition to serotonergic neurons, such as platelets, intestinal and pulmonary epithelial cells, astrocytes, spleen, kidney, uterus and placenta ${ }^{[54-56]}$. SERT is the target for several classes of antidepressant drugs including selective serotonin reuptake inhibitors and tricyclic antidepressants and drugs of abuse such as cocaine and 3,4-methylenedioxymethamphetamine (MDMA) which all inhibit SERT uptake of serotonin. As serotonylation of Rab4 also inhibits serotonin uptake into cells, alterations in monoaminylation of Rab4 could have important therapeutic consequences for drug addiction and depression. Rab4 serotonylation also regulates translocation of the GLUT4 glucose transporter to the cell membrane in skeletal muscle cells leading to increased uptake of glucose and glycogen content ${ }^{[57]}$.

The Rho family of small G-proteins regulate extracellular signaling pathways that regulate actin, cell cycle progression and gene expression ${ }^{[39]}$. The small G-protein RhoA is serotonylated and thereby rendered constitutively active during platelet activation and aggregation ${ }^{[1]}$. In vascular smooth muscle cells, serotonin induces serotonylation of RhoA leading to its activation and then proteasomal degradation ${ }^{[4,58]}$. Serotonylation of RhoA in these smooth muscle cells is dependent on uptake of serotonin into the cells by SERT and stimulation of $5-\mathrm{HT} 2 \mathrm{~A}$ receptors on the cell membrane ${ }^{[44]}$ likely needed to increase local $\mathrm{Ca}^{2+}$ concentrations to allow TG2 to assume the open conformation. Serotonylation of RhoA is increased in pulmonary smooth muscle cells, platelets and lung in patients with pulmonary hypertension suggesting that serotonylation of RhoA is a novel target for treatment ${ }^{[44]}$. Retinoic acid-induced neuronal differentiation of the SH-SY5Y neuroblastoma cell line is dependent on transamidation and activation of RhoA ${ }^{[59]}$. However, from this report it is not clear whether monoamines or polyamines were bound to RhoA, leading to increased activation of RhoA.

Other members of the Rho family of small G-proteins have also been reported. There is an increase in serotonylation or monoaminylation of Rac1 and Cdc42 but not RhoA in neuronal cells following stimulation of several different receptor systems capable of increasing local $\mathrm{Ca}^{2+}$ concentrations ${ }^{[24,25,60]}$. The increase in local $\mathrm{Ca}^{2+}$ is needed for TG2-dependent transamidation as previously discussed. Stimulation of $5-\mathrm{HT} 2 \mathrm{~A} / \mathrm{C}$ receptors results in a monoaminylation-dependent increase in the size of dendritic spines in primary cortical neurons in culture and is likely to take place after stimulation of other receptors systems that increase local $\mathrm{Ca}^{2+}$ concentrations ${ }^{[24]}$. This finding is consistent with the known ability of Rac1 and Cdc42 to regulate the actin cytoskeleton in dendritic spines and dendritic spine formation and morphology ${ }^{[61]}$. Regulation of dendritic spine morphology plays an important role in memory and learning and is perturbed in several psychiatric and neurological diseases ${ }^{[62]}$. A further understanding of the role of monoaminylation in the regulation of dendritic spines could inform novel treatment approaches for these diseases. 
Ras is the only member of the Ras family of small G-proteins known to be monoaminylated. Ras was shown to be a target for serotonylation using a novel mass spectrometry and in vivo tagging approach with a serotonin analog as well as serotonin ${ }^{[49]}$. The switch 2 glutamine in Ras is necessary for GAP-catalyzed GTP hydrolysis ${ }^{[48]}$ suggesting that serotonylation would lead to constitutive activation and indeed this was shown to be the case ${ }^{[49]}$.

Similar to the small G-proteins, two alpha subunits of heterotrimeric G-proteins have also been found to be monoaminylated. Gaq and Gao1 are monoaminylated at glutamine residues in the catalytic core of the proteins, specifically Q205 and Q209 respectively, resulting in their constitutive activation ${ }^{[36]}$.

Fibronectin and other extra-cellular proteins are substrates for serotonylation and other forms of monoaminylation. For example, fibronectin and cell surface proteins are serotonylated in C6 glioma cell cultures resulting in aggregation of extracellular proteins adjacent to cells ${ }^{[63]}$. Other monoamines, specifically dopamine and norepinephrine, are transamidated to fibronectin each resulting in similar effects of protein aggregation ${ }^{[37]}$. These monoamines compete for the same eleven binding sites but have different affinities for them. In osteoblast cultures, fibronectin can be serotonylated ${ }^{[64]}$. Serotonylation appears to compete with protein cross-linking of fibronectin resulting in reduced collagen deposition and delayed mineralization. Interestingly, the TG factor XIIla rather than TG2 catalyzes serotonylation of extracellular fibronectin associated with osteoblasts. Serotonylation of fibronectin as well as RhoA (as mentioned above) is found in pulmonary vascular artery smooth muscle cells and correlates with the progression of pulmonary hypertension ${ }^{[65]}$. Together these findings suggest a role for serotonylation in the pathogenesis of pulmonary hypertension and could be a target for novel treatment approaches.

In aortic vascular smooth muscle cells, monoaminylation is involved in vascular contraction. Several proteins are serotonylated including $\alpha$-actin, $\beta$-actin, $\gamma$-actin, actin-binding protein filamin $A$, and myosin heavy chain ${ }^{[6]}$. Inhibition of TG diminished isometric contractions, a process dependent on $\alpha$-actin, suggesting that monoaminylation of $\alpha$-actin is critical for this function. Similarly, monoaminylation of $\alpha$-actin with norepinephrine is necessary for norepinephrine-induced vascular contraction ${ }^{[67]}$.

\section{CONCLUSIONS}

As described in this review, TG2 can impact neurotransmitter receptor regulated-functions in the role of the alpha subunit of a heterodimeric G-protein. Alternatively, TG2 conformation and transamidation activity can be regulated by neurotransmitter systems that modulate local intracellular $\mathrm{Ca}^{2+}$ concentrations. And lastly TGs can use monoamine neurotransmitters as substrates for transamidation reactions. Each of these processes do not work in isolation but rather can interact and compete. TG2 can exist in either the closed GTP-bound form that functions as a G-protein or in the open $\mathrm{Ca}^{2+}$ bound form that catalyzes transamidation reactions. Indeed, the interplay between the regulation of receptor signaling systems that increase local levels of intracellular $\mathrm{Ca}^{2+}$ to allow TG2 to be in the open conformation capable of catalyzing transamidation oppose the function of TG2 signaling as a G-protein in the closed conformation. How this competition might play out in normal physiological systems or in pathology has yet to be explored.

There is also competition in the use of monoamine neurotransmitters as substrates for transamidation reactions. There can be competition among monoamine neurotransmitters in transamidation reactions as demonstrated by Hummerich et al. ${ }^{[37]}$. They reported that the affinity of monoamines varies for the substrate fibronectin, suggesting that the affinity and relative concentrations of the monoamines dictates which monoamine is bound to a protein. Competition can also take place between transamidation to monoamines or protein cross-linking. In osteoblasts, there is competition between serotonin and proteins as substrates for transamidation to fibronectin impacting mineralization and fibronectin deposition ${ }^{[64]}$. Competition between monoamines and proteins as substrates in other physiological and pathological situations is likely to exist and depend on substrate availability. Alterations in the availability of intracellular neurotransmitters can be regulated by either high capacity, low selectivity transporters such as the organic cation transporters and plasma membrane monoamine transporters or the low capacity, high selectivity transporters for serotonin (SERT, SLC6A4), for noradrenaline (SLC6A2) and for dopamine (SLC6A3). These transporters are highly regulated and all are likely involved in feedback mechanisms such as regulation of SERT by serotonylation of Rab4 described above ${ }^{[50]}$. Moreover, medications and illicit drugs are targets for these transporters and could thereby impact the availability of these monoamines as intracellular substrates for competition for transamidation. A better understanding of the regulation of the availability intracellular monoamines for monoaminylation and for competition with protein cross-linking will likely inform future treatment approaches for numerous disorders especially those related to monoamines such as schizophrenia, depression and anxiety.

Interaction and co-operation between the regulation of TG2 transamidation reactions by receptor-regulated $\mathrm{Ca}^{2+}$ concentrations and 
monoaminylation has also been demonstrated. Serotonylation of Rac1 and Cdc42 is regulated by the serotonin receptors $5-\mathrm{HT} 2 \mathrm{~A}$ and $5-\mathrm{HT} 2 \mathrm{C}$ as well as muscarinic and NMDA receptors in neurons ${ }^{[24,60]}$. Similarly, 5-HT2A receptor stimulation results in serotonylation of Rab4 and RhoA in platelets ${ }^{[1]}$.

From this review, we can see that TGs are important regulators of a plethora of receptor and neurotransmitter regulated processes. These TG-mediated processes range from modulation of dendritic spine morphology impacting learning and memory functions, to depression, blood clot formation, and hypertension. We are only beginning to appreciate the mechanisms by which TG signal transduction impacts normal physiology and can be targeted for treatment in disease states. New

\section{REFERENCES}

1. Walther DJ, Peter JU, Winter S, Höltje M, Paulmann N, Grohmann M, Vowinckel J, AlamoBethencourt V, Wilhelm CS, Ahnert-Hilger G, Bader M. Serotonylation of small GTPases is a signal transduction pathway that triggers platelet alphagranule release. Cell. 2003; 115(7): 851-862.

2. Pisano JJ, Finlayson JS, Peyton MP. [Cross-link in fibrin polymerized by factor 13: epsilon(gamma-glutamyl)lysine]. Science. 1968; 160(830): 892-893.

3. Griffin M, Casadio R, Bergamini CM. Transglutaminases: nature's biological glues. Biochem J. 2002; 368(Pt 2): 377-396.

4. Birckbichler PJ, Orr GR, Conway E, Patterson MK, Jr. Transglutaminase activity in normal and transformed cells. Cancer Res. 1977; 37(5): 1340-1344.

5. Dieterich W, Ehnis T, Bauer M, Donner P, Volta $U$, Riecken EO, Schuppan D. Identification of tissue transglutaminase as the autoantigen of celiac disease. Nat Med. 1997; 3(7): 797-801.

6. Grenard P, Bates MK, Aeschlimann D. Evolution of transglutaminase genes: identification of a transglutaminase gene cluster on human chromosome 15q15. Structure of the gene encoding transglutaminase $X$ and a novel gene family member, transglutaminase $Z$. J Biol Chem. 2001; 276(35): 33066-33078.

7. Mycek MJ, Waelsch $\mathrm{H}$. The enzymatic deamidation of proteins. J Biol Chem. 1960; 235: 3513-3517.

8. Lorand L, Graham RM. Transglutaminases: crosslinking enzymes with pleiotropic functions. Nat Rev Mol Cell Biol. 2003; 4(2): 140-156. treatment approaches will likely result from a better understanding of the role of TGs, especially TG2, in receptor and neurotransmitter-regulated functions.

\section{CONFLICTS OF INTEREST}

The author declares that there is no conflict of interest regarding the publication of this paper.

\section{ACKNOWLEDGEMENT}

Funding was provided by the National Institutes for Health R01NS088059.

9. Tatsukawa H, Furutani Y, Hitomi K, Kojima S. Transglutaminase 2 has opposing roles in the regulation of cellular functions as well as cell growth and death. Cell Death Dis. 2016; 7(6): e2244.

10. Achyuthan KE, Greenberg CS. Identification of a guanosine triphosphate-binding site on guinea pig liver transglutaminase. Role of GTP and calcium ions in modulating activity. J Biol Chem. 1987; 262(4): 1901-1906.

11. Nakaoka H, Perez DM, Baek KJ, Das T, Husain A, Misono K, Im MJ, Graham RM. Gh: A GTP-binding protein with transglutaminase activity and receptor signaling function. Science. 1994; 264: 1593-1596.

12. Pinkas DM, Strop P, Brunger AT, Khosla $C$. Transglutaminase 2 undergoes a large conformational change upon activation. PLoS Biol. 2007; 5(12): e327.

13. Feng JF, Rhee SG, Im MJ. Evidence that phospholipase delta1 is the effector in the $\mathrm{Gh}$ (transglutaminase II)-mediated signaling. J Biol Chem. 1996; 271(28): 16451-16454.

14. Baek KJ, Kwon NS, Lee HS, Kim MS, Muralidhar $\mathrm{P}, \mathrm{Im}$ MJ. Oxytocin receptor couples to the $80 \mathrm{kDa}$ Gh alpha family protein in human myometrium. Biochem J. 1996; 315(Pt 3): 739-744.

15. Park ES, Won JH, Han KJ, Suh PG, Ryu SH, Lee HS, Yun HY, Kwon NS, Baek KJ. Phospholipase C-delta1 and oxytocin receptor signalling: evidence of its role as an effector. Biochem J. 1998; 331(Pt 1): 283-289.

16. Vezza R, Habib A, FitzGerald GA. Differential signaling by the thromboxane receptor isoforms 
via the novel GTP-binding protein, Gh. J Biol Chem. 1999; 274(18): 12774-12779.

17. Lin YF, Tseng MJ, Hsu HL, Wu YW, Lee YH, Tsai YH. A Novel Follicle-Stimulating HormoneInduced Gah/Phospholipase C- $\delta 1$ Signaling Pathway Mediating Rat Sertoli Cell $\mathrm{Ca}^{2+}$-Influx. Mol Endocrinol. 2006; 20(10): 2514-2527.

18. Feng JF, Readon M, Yadav SP, Im MJ. Calreticulin Down-Regulates both GTP Binding and Transglutaminase Activities of Transglutaminase II. Biochemistry. 1999; 38(33): 10743-10749.

19. Baek KJ, Das T, Gray CD, Desai S, Hwang KC, Gacchui R, Ludwig M, Im MJ. A 50 KDa protein modulates guanine nucleotide binding of transglutaminase II. Biochemistry. 1996; 35(8): 2651-2657.

20. Smethurst PA, Griffin M. Measurement of tissue transglutaminase activity in a permeabilized cell system: its regulation by $\mathrm{Ca}^{2+}$ and nucleotides. Biochem J. 1996; 313(Pt 3): 803-808.

21. Clapham DE. Calcium signaling. Cell. 2007; 131(6): 1047-1058.

22. Kiraly R, Demeny M, Fesus L. Protein transamidation by transglutaminase 2 in cells: a disputed $\mathrm{Ca}^{2+}$-dependent action of a multifunctional protein. FEBS J. 2011; 278(24): 4717-4739.

23. Salter NW, Ande SR, Nguyen HK, Nyomba $\mathrm{BL}$, Mishra S. Functional characterization of naturally occurring transglutaminase 2 mutants implicated in early-onset type 2 diabetes. J Mol Endocrinol. 2012; 48(3): 203-216.

24. Mi Z, Si T, Kapadia K, Li Q, Muma NA. Receptorstimulated transamidation induces activation of Rac1 and Cdc42 and the regulation of dendritic spines. Neuropharmacology. 2017; 117: 93-105.

25. Dai Y, Dudek NL, Li Q, Muma NA. Phospholipase $\mathrm{C}, \mathrm{Ca}^{2+}$, and calmodulin signaling are required for 5-HT2A receptor-mediated transamidation of Rac1 by transglutaminase. Psychopharmacology (Berl). 2011; 213(2-3): 403-412.

26. Zhang J, Lesort M, Guttmann RP, Johnson GV. Modulation of the in situ activity of tissue transglutaminase by calcium and GTP. J Biol Chem. 1998; 273(4): 2288-2295.

27. Dai Y, Dudek NL, Patel TB, Muma NA. Transglutaminase-catalyzed transamidation: a novel mechanism for Rac1 activation by 5-hydroxytryptamine2A receptor stimulation. J Pharmacol Exp Ther. 2008; 326(1): 153-162.
28. Vyas FS, Nelson CP, Freeman F, Boocock DJ, Hargreaves AJ, Dickenson JM. beta2-adrenoceptorinduced modulation of transglutaminase 2 transamidase activity in cardiomyoblasts. Eur J Pharmacol. 2017; 813: 105-121.

29. Vyas FS, Hargreaves AJ, Bonner PLR, Boocock DJ, Coveney C, Dickenson JM. $A_{1}$ adenosine receptor-induced phosphorylation and modulation of transglutaminase 2 activity in $\mathrm{H} 9 \mathrm{c} 2$ cells: A role in cell survival. Biochem Pharmacol. 2016; 107: 41-58.

30. Algarni AS, Hargreaves AJ, Dickenson JM. Role of transglutaminase 2 in $\mathrm{PAC}_{1}$ receptor mediated protection against hypoxia-induced cell death and neurite outgrowth in differentiating N2a neuroblastoma cells. Biochem Pharmacol. 2017; 128: 55-73.

31. Mishra S, Murphy LJ. Phosphorylation of transglutaminase 2 by PKA at Ser216 creates 14-3-3 binding sites. Biochem Biophys Res Commun. 2006; 347(4): 1166-1170.

32. Mishra S, Melino G, Murphy LJ. Transglutaminase 2 kinase activity facilitates protein kinase A-induced phosphorylation of retinoblastoma protein. J Biol Chem. 2007; 282(25): 18108-18115.

33. Pavlyukov MS, Antipova NV, Balashova MV, Shakhparonov MI. Detection of Transglutaminase 2 conformational changes in living cell. Biochem Biophys Res Commun. 2012; 421(4): 773-779.

34. Stabile LP, Lyker JS, Gubish CT, Zhang W, Grandis JR, Siegfried JM. Combined targeting of the estrogen receptor and the epidermal growth factor receptor in non-small cell lung cancer shows enhanced antiproliferative effects. Cancer Res. 2005; 65(4): 1459-1470.

35. Dale GL, Friese $P$, Batar P, Hamilton SF, Reed $\mathrm{GL}$, Jackson KW, Clemetson KJ, Alberio L. Stimulated platelets use serotonin to enhance their retention of procoagulant proteins on the cell surface. Nature. 2002; 415(6868): 175-179.

36. Vowinckel J, Stahlberg S, Paulmann N, Bluemlein K, Grohmann M, Ralser M, Walther DJ. Histaminylation of glutamine residues is a novel posttranslational modification implicated in G-protein signaling. FEBS Lett. 2012; 586(21): 3819-3824.

37. Hummerich R, Thumfart JO, Findeisen P, Bartsch D, Schloss P. Transglutaminasemediated transamidation of serotonin, dopamine and noradrenaline to fibronectin: evidence for a general mechanism of monoaminylation. FEBS Lett. 2012; 586(19): 3421-3428. 
38. Muma NA, Mi Z. Serotonylation and Transamidation of Other Monoamines. ACS Chem Neurosci. 2015; 6(7): 961-969.

39. Wennerberg K, Rossman KL, Der CJ. The Ras superfamily at a glance. J Cell Sci. 2005; 118(5): 843-846.

40. Hakoshima T. Structural basis of the Rho GTPase signaling. J Biochem. 2003; 134(3): 327-331.

41. Flatau G, Landraud L, Boquet P, Bruzzone M, Munro P. Deamidation of RhoA glutamine 63 by the Escherichia coli CNF1 toxin requires a short sequence of the GTPase switch 2 domain. Biochem Biophys Res Commun. 2000; 267(2): 588-592.

42. Lerm M, Selzer J, Hoffmeyer A, Rapp UR, Aktories K, Schmidt G. Deamidation of Cdc42 and Rac by Escherichia coli cytotoxic necrotizing factor 1: activation of c-Jun N-terminal kinase in HeLa cells. Infect Immun. 1999; 67(2): 496-503.

43. Schmidt G, Goehring UM, Schirmer J, Lerm $\mathrm{M}$, Aktories K. Identification of the C-terminal part of Bordetella dermonecrotic toxin as a transglutaminase for rho GTPases. J Biol Chem. 1999; 274(45): 31875-31881.

44. Guilluy C, Eddahibi S, Agard C, Guignabert C, Izikki M, Tu L, Savale L, Humbert M, Fadel E, Adnot S, Loirand G, Pacaud P. RhoA and Rho kinase activation in human pulmonary hypertension: role of 5-HT signaling. Am J Respir Crit Care Med. 2009; 179(12): 1151-1158.

45. Doye A, Mettouchi A, Bossis G, Clément R, Buisson-Touati C, Flatau G, Gagnoux L, Piechaczyk M, Boquet $P$, Lemichez E. CNF1 exploits the ubiquitin-proteasome machinery to restrict Rho GTPase activation for bacterial host cell invasion. Cell. 2002; 111(4): 553-564.

46. Masuda M, Minami M, Shime $H$, Matsuzawa $T$, Horiguchi Y. In vivo modifications of small GTPase Rac and Cdc42 by Bordetella dermonecrotic toxin. Infect Immun. 2002; 70(2): 998-1001.

47. Horiguchi $Y$, Inoue N, Masuda M, Kashimoto T, Katahira J, Sugimoto N, Matsuda M. Bordetella bronchiseptica dermonecrotizing toxin induces reorganization of actin stress fibers through deamidation of GIn-63 of the GTP-binding protein Rho. Proc Natl Acad Sci U S A. 1997; 94(21): 11623-11626.

48. Cherfils J, Zeghouf M. Regulation of Small GTPases by GEFs, GAPs, and GDIs. Physiol Rev. 2013; 93(1): 269-309.

49. Lin JC, Chou CC, Gao S, Wu SC, Khoo KH, Lin $\mathrm{CH}$. An in vivo tagging method reveals that Ras undergoes sustained activation upon transglutaminase-mediated protein serotonylation. Chembiochem. 2013; 14(7): 813-817.
50. Ahmed BA, Jeffus BC, Bukhari SI, Harney JT, Unal R, Lupashin VV, van der Sluijs P, Kilic F. Serotonin transamidates Rab4 and facilitates its binding to the $\mathrm{C}$ terminus of serotonin transporter. J Biol Chem. 2008; 283(14): 9388-9398.

51. Paulmann N, Grohmann M, Voigt JP, Bert B, Vowinckel J, Bader M, Skelin M, Jevsek M, Fink $\mathrm{H}$, Rupnik M, Walther DJ. Intracellular serotonin modulates insulin secretion from pancreatic beta-cells by protein serotonylation. PLoS Biol. 2009; 7(10): e1000229.

52. Bernassola F, Federici M, Corazzari M, Terrinoni A, Hribal ML, De Laurenzi V, Ranalli M, Massa O, Sesti G, McLean WH, Citro G, Barbetti F, Melino $\mathrm{G}$. Role of transglutaminase 2 in glucose tolerance: knockout mice studies and a putative mutation in a MODY patient. FASEB J. 2002; 16(11): 1371-1378.

53. Iismaa SE, Aplin M, Holman S, Yiu TW, Jackson K, Burchfield JG, Mitchell CJ, O'Reilly L, Davenport A, Cantley J, Schmitz-Peiffer C, Biden TJ, Cooney GJ, Graham RM. Glucose Homeostasis in Mice Is Transglutaminase 2 Independent. PLoS One. 2013; 8(5): e63346.

54. Hoffman BJ, Mezey E, Brownstein MJ. Cloning of a serotonin transporter affected by antidepressants. Science. 1991; 254(5031): 579-580.

55. Kimelberg HK, Katz DM. High-affinity uptake of serotonin into immunocytochemically identified astrocytes. Science. 1985; 228(4701): 889-891.

56. Lesch KP, Wolozin BL, Murphy DL, Reiderer P. Primary structure of the human platelet serotonin uptake site: identity with the brain serotonin transporter. J Neurochem. 1993; 60(6): 2319-2322.

57. Al-Zoairy R, Pedrini MT, Khan MI, Engl J, Tschoner A, Ebenbichler C, Gstraunthaler G, Salzmann K, Bakry R, Niederwanger A. Serotonin improves glucose metabolism by Serotonylation of the small GTPase Rab4 in L6 skeletal muscle cells. Diabetol Metab Syndr. 2017; 9: 1.

58. Guilluy C, Rolli-Derkinderen M, Tharaux PL, Melino G, Pacaud P, Loirand G. Transglutaminasedependent RhoA activation and depletion by serotonin in vascular smooth muscle cells. J Biol Chem. 2007; 282(5): 2918-2928.

59. Singh US, Pan J, Kao YL, Joshi S, Young KL, Baker KM. Tissue Transglutaminase Mediates Activation of RhoA and MAP Kinase Pathways during Retinoic Acid-induced Neuronal Differentiation of SH-SY5Y Cells. J Biol Chem. 2003; 278(1): 391-399.

60. Dai Y, Dudek NL, Patel TB, Muma NA. Transglutaminase-catalyzed Transamidation: A 
Novel Mechanism for Rac1 Activation by 5-HT2A Receptor Stimulation. J Pharmacol Exp Ther. 2008; 326(1): 153-162.

61. Martino A, Ettorre M, Musilli M, Lorenzetto E, Buffelli M, Diana G. Rho GTPase-dependent plasticity of dendritic spines in the adult brain. Front Cell Neurosci. 2013; 7: 62.

62. Penzes $P$, Cahill ME, Jones KA, VanLeeuwen JE, Woolfrey KM. Dendritic spine pathology in neuropsychiatric disorders. Nat Neurosci. 2011; 14(3): 285-293.

63. Hummerich R, Schloss P. Serotonin--more than a neurotransmitter: transglutaminase-mediated serotonylation of C6 glioma cells and fibronectin Neurochem Int. 2010; 57(1): 67-75.

64. Cui C, Kaartinen MT. Serotonin (5-HT) inhibits Factor XIII-A-mediated plasma fibronectin matrix assembly and crosslinking in osteoblast cultures via direct competition with transamidation. Bone. 2015; 72: 43-52.

65. Liu Y, Wei L, Laskin DL, Fanburg BL. Role of protein transamidation in serotonin-induced proliferation and migration of pulmonary artery smooth muscle cells. Am J Respir Cell Mol Biol. 2011; 44(4): 548-555.

66. Watts SW, Priestley JR, Thompson JM. Serotonylation of vascular proteins important to contraction. PLoS One. 2009; 4(5): e5682.

67. Johnson KB, Thompson JM, Watts SW. Modification of proteins by norepinephrine is important for vascular contraction. Frontiers in physiology. 2010; 1: 131. 